\title{
CONGENITAL, MALFORMATIONS
}

\section{MANAGEMENT OF CRANIOSYNOSTOSIS}

The safety and efficacy of early treatment of craniosynostosis, using minimally invasive endoscopic strip craniectomies, were evaluated prospectively in 100 patients (72 boys, 28 girls) treated at the University of Missouri Hospital, Columbus, MO. Ages ranged between 2 weeks and 9.5 months (mean 3.1 months); 63 were treated under 16 weeks of age. Of 106 stenosed sutures, 61 were sagittal, 23 coronal, 18 metopic, and 4 lambdoid. After surgery, all patients were fitted with custom-made molding helmets worn for up to 7 months. Follow-up ranged from 4 to 50 months. Mean surgical operative time was 52.7 minutes, and mean estimated blood loss was $26.2 \mathrm{ml}$. One patient required an intraoperative blood transfusion and 9 required postoperative transfusions. All except 3 patients were discharged on the first postoperative day, and without significant complication. This minimaliy invasive method of treatment is safe and effective and superior to the current more extensive surgical procedures. The optimal time for operation is under 6 months, preferably at 3 months. The majority achieve normocephaly or significant correction. (Jimenez DF, Barone CM, Cartwright CC, Baker L. Early management of craniosynostosis using endoscopic-assisted strip craniectomies and cranial orthotic molding therapy. Pediatrics July 2002;110:97-104). (Reprints: David F Jimenez MD, Division of Neurological Surgery, University Hospital and Clinics, One Hospital Drive, Columbia, MO 65212).

COMMENT. Sagittal synostosis has a reported incidence of 1 in 1000 live births. Coronal and metopic forms are less common and lambdoid craniosynostosis is rare. Optimal time for operation recommended for sagittal synostosis is usually at 3 to 4 months, and later for the other varieties. The less invasive method described by Jimenez et al appears safe and effective when employed at an early age. However, as pointed out by Maugans TA in an editorial (The misshapen head. Pediatrics July 2002;110:166-167), the minimal surgery is followed by an absolute requirement of weeks to months of cranial orthosis use. In the more extensive cranial remodeling surgery, optimal cosmetic results are realized immediately after postoperative swelling subsides and helmets are not usually required.

\section{SEIZURE DISORDERS}

\section{INTERLEUKIN GENE MARKER FOR FEBRILE SEIZURES}

The use of polymorphisms for interleukin 1B (IL-1B) exon 5 and interleukin 1 receptor antagonist (IL-1Ra) as markers of susceptibility to febrile seizures was evaluated in Taiwanese children (group 1: 51 with febrile convulsions and group 2: 83 normal controls) at China Medical College Hospital, Taichung, Taiwan. Genotype proportions and allele frequencies in the 2 groups were not significantly different for IL-1B exon 5, but were significantly different for IL-1Ra. Frequencies of alleles I and II for IL-1Ra were $100(98 \%)$ and 2 (2\%) in group 1 and $152(91.6 \%)$ and $14(8.4 \%)$ in group 2. The IL-1 Ra allele I is associated with a higher susceptibility to febrile convulsions. (Tsai F-J, Hsieh Y-Y, Chang C$\mathrm{C}$, et al. Polymorphisms for interleukein $1 \mathrm{~B}$ exon 5 and interleukin 1 receptor antagonist in Taiwanese children with febrile convulsions. Arch Pediatr Adolesc Med June 2002;156:545-548). (Reprints: Fuu-Jen Tsai MD PhD, Department of Pediatrics and Medical Genetics, China Medical College Hospital, 2 Yuh-Der Rd, Taichung Taiwan). 